\title{
Intracranial Benign Fibrous Histiocytoma
}

National Cancer Institute

\section{Source}

National Cancer Institute. Intracranial Benign Fibrous Histiocytoma. NCI Thesaurus.

Code C129549.

A benign fibrous histiocytoma involving the dura or cranial bone. 